Article

\title{
Some Characterizations of Generalized Null Scrolls
}

\author{
Jinhua Qian ${ }^{1} * \mathbb{D}$, Xueshan Fu ${ }^{2}$ and Seoung Dal Jung ${ }^{2}$ \\ 1 Department of Mathematics, Northeastern University, Shenyang 110004, China \\ 2 Department of Mathematics, Jeju National University, Jeju 690-756, Korea; 1600090@stu.neu.edu.cn (X.F.); \\ sdjung@jejunu.ac.kr (S.D.J.) \\ * Correspondence: qianjinhua@mail.neu.edu.cn; Tel.: +86-138-8935-7350
}

Received: 9 September 2019; Accepted: 1 October 2019; Published: 10 October 2019

\begin{abstract}
In this work, a family of ruled surfaces named generalized null scrolls in Minkowski 3-space are investigated via the defined structure functions. The relations between the base curve and the ruling flow of the generalized null scroll are revealed. The Gaussian curvature, mean curvature, second Gaussian curvature and the second mean curvature are given and related to each other. Last but not least, the generalized null scrolls whose base curves are k-type null helices are discussed and several examples are presented.
\end{abstract}

Keywords: ruled surface; generalized null scroll; null curve; k-type null helix; structure function

\section{Introduction}

In the modern surface model systems, the immersed surfaces swept out by a straight line moving along a curve play an important role, which are the simplest foliated submanifolds and called ruled submanifolds. Such submanifolds can be applied in physics, motion analysis, manufacturing of products, designing cars, ships and many other model-based objects and so on. For a view of geometry, ruled submanifolds are essential for studying kinematical and positional spatial mechanisms.

Due to their particular geometry properties and structures, ruled submanifolds are widely discussed from different viewpoints not only in Euclidean space but also in Minkowski space. In Minkowski space, the ruled submanifolds have been divided into several kinds according to the causal characters of the base curves and the ruling flows [1,2]. Among of them, the ruled submanifolds with lightlike rulings are very particular because the norm of lightlike vector vanishes everywhere. In Minkowski 3-space, if the base curve is a lightlike curve, then the ruled surface with lightlike ruling is called a null scroll which has been studied by many geometers [3-5]. Furthermore, the null scroll under a Cartan Frenet frame is called a B-scroll [6]. In 2006, Kim, Y. H. [7] extended the B-scroll in Minkowski 3-space into higher dimensional Minkowski space, which is then called a generalized B-scroll and the properties of such surfaces are studied through its Gauss map. In 2015, one of the authors proposed the concept of the structure function for null curves [8], which has been regarded as a useful research tool for the problems related to null curves. Motivated by this idea, in this paper, we define a new kind of ruled surfaces named the generalized null scrolls (GNS) by omitting the normalized constraint of transversality of null scrolls, and we focus on the geometric properties of GNS via the new defined structure functions.

The paper is organized as follows. In Section 2, some basic facts including the Frenet formulae and the structure function of null curves are reviewed, then a new surface GNS is defined based on the concept of the null scrolls. The geometric properties such as the Gaussian curvature, mean curvature, second Gaussian curvature and the second mean curvature of GNS are shown and related by the new defined structure functions of GNS together with the distribution parameter in Section 3. Last, the generalized null scrolls whose base curves are k-type null helices are characterized and several examples are presented explicitly. 
Throughout this paper, we assume that all the geometric objects under consideration are smooth and all surfaces are connected unless otherwise stated.

\section{Preliminaries}

Let $\mathbb{E}_{1}^{3}$ be the Minkowski 3-space with natural Lorentzian metric

$$
\langle\cdot, \cdot\rangle=d x_{1}^{2}+d x_{2}^{2}-d x_{3}^{2}
$$

in terms of the natural coordinate system $\left(x_{1}, x_{2}, x_{3}\right)$. A vector $v$ in $\mathbb{E}_{1}^{3}$ is said to be spacelike, timelike and lightlike (null) if $\langle v, v\rangle>0$ or $v=0,\langle v, v\rangle\langle 0$ and $\langle v, v\rangle=0(v \neq 0)$, respectively. The norm of a vector $v$ is defined by $\|v\|=\sqrt{|\langle v, v\rangle|}$. An arbitrary curve $\gamma$ is spacelike, timelike or lightlike if its tangent vector $\gamma^{\prime}$ is spacelike, timelike or lightlike, correspondingly. At the same time, a surface is said to be timelike, spacelike or lightlike if its normal vector is spacelike, timelike or lightlike, respectively.

Let $a(s): \mathrm{I}_{1} \rightarrow \mathbb{E}_{1}^{3}$ be a curve, where $\mathrm{I}_{1}$ is an open interval on a real line $\mathbb{R}$ and $b(s)$ a transversal vector field along $a(s)$. Then the immersion $X$ parameterized by

$$
X(s, t)=a(s)+t b(s), \quad\left(t \in \mathrm{I}_{2} \subset \mathbb{R}\right)
$$

is called a ruled surface with base curve $a(s)$ and ruling flow $b(s)$. Such kinds of surfaces in $\mathbb{E}_{1}^{3}$ have been classified into five types according to the causal character of the base curve and the ruling flow (for the details see [2]). Among of them, for the ruled surfaces with lightlike rulings, the base curves can be null curves or non-null curves obviously. However, the ruled surfaces with non-null base curves are equivalent to the ones with null base curves via the appropriate method as stated in [3], at the same time, the normalization condition $\left\langle a^{\prime}(s), b(s)\right\rangle=1$ should be satisfied. Therefore, without loss of generality, we always can choose a null curve as the base curve of a ruled surface with lightlike ruling and $\left\langle a^{\prime}(s), b(s)\right\rangle=1$ is satisfied in $\mathbb{E}_{1}^{3}$.

Proposition 1 ([8]). Let a (s) be a null curve parameterized by the null arc length s (i.e., $\left.\left\|a^{\prime \prime}(s)\right\|=1\right)$ in $\mathbb{E}_{1}^{3}$. Then there exists a unique Cartan frame $\left\{a^{\prime}(s)=T(s), N(s), B(s)\right\}$ such that

$$
\left(\begin{array}{c}
T^{\prime}(s) \\
N^{\prime}(s) \\
B^{\prime}(s)
\end{array}\right)=\left(\begin{array}{ccc}
0 & 1 & 0 \\
\kappa(s) & 0 & -1 \\
0 & -\kappa(s) & 0
\end{array}\right)\left(\begin{array}{c}
T(s) \\
N(s) \\
B(s)
\end{array}\right)
$$

where $\langle T(s), T(s)\rangle=\langle B(s), B(s)\rangle=\langle T(s), N(s)\rangle=\langle B(s), N(s)\rangle=0,\langle T(s), B(s)\rangle=\langle N(s), N(s)\rangle=1$. And

$$
T(s) \times B(s)=N(s), \quad N(s) \times T(s)=T(s), \quad N(s) \times B(s)=-B(s) .
$$

In the sequel, $T(s), N(s)$ and $B(s)$ is called the tangent, principal normal and binormal vector field of $a(s)$, respectively. From (1), it is easy to know that $\kappa(s)=-\frac{1}{2}\left\langle a^{\prime \prime \prime}(s), a^{\prime \prime \prime}(s)\right\rangle$. The function $\kappa(s)$ is called the null curvature of $a(s)$ which is an invariant under Lorentzian transformations $[9,10]$. Hence, a null curve is only determined by its null curvature.

At the same time, for a null curve $a(s)$ with null curvature $\kappa(s)$, we can find a function $g=g(s)$ which is related to the null curvature as

$$
\kappa(s)=\frac{1}{2}\left[\left(\log g^{\prime}\right)^{\prime}\right]^{2}-\left(\log g^{\prime}\right)^{\prime \prime}
$$

and is called the structure function of $a(s)$ [8]. In terms of the structure function $g$, the representation formulae for a null curve can be given as follows: 
Proposition 2 ([8]). Let $a(s)$ be a null curve parameterized by null arc length parameter $s$ in $\mathbb{E}_{1}^{3}$. Then it can be represented as

$$
a(s)=\int \frac{g}{2 g^{\prime}}\left(g-g^{-1}, 2, g+g^{-1}\right) d s,
$$

where $g=g(s)$ is the structure function of $a(s)$.

Definition 1 ([2]). Let $a(s): \mathrm{I}_{1} \rightarrow \mathbb{E}_{1}^{3}$ be a null curve parameterized by null arc length, and $b(s)$ a transversal null vector field along $a(s)$. Then the immersion

$$
X(s, t)=a(s)+t b(s), \quad\left(t \in \mathrm{I}_{2} \subset \mathbb{R}\right)
$$

is called a null scroll, which satisfies $\left\langle a^{\prime}(s), a^{\prime}(s)\right\rangle=0,\langle b(s), b(s)\rangle=0$ and $\left\langle a^{\prime}(s), b(s)\right\rangle=1$.

Remark 1 ([6]). In particular, a null scroll is said to be a B-scroll if its base curve a $(s)$ is under Cartan Frenet frame $\{T(s), N(s), B(s)\}$ and $b(s)=B(s)$.

Next, we talk about a more general case based on the concept of null scrolls by omitting the normalization conditions of null scrolls, i.e., $\left\langle a^{\prime}(s), b(s)\right\rangle=1$.

Definition 2. Let $a(s): \mathrm{I}_{1} \rightarrow \mathbb{E}_{1}^{3}$ be a null curve parameterized by null arc length, $b(s)$ a transversal null vector field along $a(s)$. Then the immersion parameterized by

$$
X(s, t)=a(s)+t b(s), \quad\left(t \in \mathrm{I}_{2} \subset \mathbb{R}\right)
$$

is called a generalized null scroll, which satisfies $\left\langle a^{\prime}(s), a^{\prime}(s)\right\rangle=0$ and $\langle b(s), b(s)\rangle=0$.

Remark 2. For convenience, we abbreviate the generalized null scroll as GNS.

It is well-known that a ruled surface is said to be $\operatorname{developable~if~} \operatorname{det}\left(a^{\prime}(s), b(s), b^{\prime}(s)\right)=0$. Throughout this article, we consider non-developable ruled surfaces, i.e., skew ruled surfaces. Then, the striction curve and the distribution parameter for a non-developable GNS $X$ can be well-defined. Geometrically, the striction curve is the locus of the central point which is the foot of the common perpendicular on the main ruling, it can be defined by [11]

$$
r(s)=a(s)-\frac{\left\langle a^{\prime}(s), b^{\prime}(s)\right\rangle}{\left\langle b^{\prime}(s), b^{\prime}(s)\right\rangle} b(s) .
$$

At the same time, the distribution parameter $\varphi=\varphi(s)$ of $X$ can be arrived as [11]

$$
\varphi(s)=\frac{\operatorname{det}\left(a^{\prime}(s), b(s), b^{\prime}(s)\right)}{\left\langle b^{\prime}(s), b^{\prime}(s)\right\rangle} .
$$

It is clear that $\varphi(s)$ vanish nowhere for a non-developable GNS X. Without loss of generality, we may choose the striction curve as the base curve of $X$, i.e., $\left\langle a^{\prime}(s), b^{\prime}(s)\right\rangle=0$ throughout this paper.

\section{The Geometric Properties of GNS}

Let $X(s, t)=a(s)+t b(s)$ be a GNS in $\mathbb{E}_{1}^{3}$ and $s$ the null arc length parameter of the base curve $a(s)$. Since $b(s)$ is a transversal vector along $a(s)$, then it can be decomposed by the Cartan frame $\{T(s), N(s), B(s)\}$ of $a(s)$ as

$$
b(s)=\lambda(s) T(s)+\mu(s) N(s)+\omega(s) B(s)
$$


For some smooth functions $\lambda=\lambda(s), \mu=\mu(s)$ and $\omega=\omega(s)$, from Proposition 1 , it is easy to get

$$
b^{\prime}(s)=\left(\lambda^{\prime}+\mu \kappa\right) T(s)+\left(\mu^{\prime}+\lambda-\omega \kappa\right) N(s)+\left(\omega^{\prime}-\mu\right) B(s) .
$$

Based on the assumption that $a(s)$ is the striction curve, i.e., $\left\langle a^{\prime}(s), b^{\prime}(s)\right\rangle=0$, together with (7), we have $\mu=\omega^{\prime}$. Hence, we have from (6)

$$
b(s)=\lambda(s) T(s)+\omega^{\prime}(s) N(s)+\omega(s) B(s) .
$$

Furthermore, since $\langle b(s), b(s)\rangle=0$, from (8), we have $\omega^{\prime 2}+2 \lambda \omega=0$. Assume $\omega=0$, then $b(s)=\lambda(s) T(s)$, i.e., $X$ is developable. Hence $\omega \neq 0$ and

$$
\lambda=-\frac{\omega^{\prime 2}}{2 \omega} \text {. }
$$

Thus, $b(s)$ and $b^{\prime}(s)$ can be rewritten as

$$
b(s)=-\frac{\omega^{\prime 2}}{2 \omega} T(s)+\omega^{\prime} N(s)+\omega B(s)
$$

and

$$
b^{\prime}(s)=\left(\frac{\omega^{\prime 3}}{2 \omega^{2}}-\frac{\omega^{\prime} \omega^{\prime \prime}}{\omega}+\omega^{\prime} \kappa\right) T(s)+\left(\omega^{\prime \prime}-\frac{\omega^{\prime 2}}{2 \omega}-\omega \kappa\right) N(s) .
$$

Therefore, we obtain

$$
\left\langle b^{\prime}, b^{\prime}\right\rangle=\left(\omega^{\prime \prime}-\frac{\omega^{\prime 2}}{2 \omega}-\omega \kappa\right)^{2}
$$

From (1), (10)-(12), the distribution parameter $\varphi(s)$ in (5) of $X$ is given by

$$
\varphi(s)=\frac{\omega}{f}
$$

where

$$
f=f(s)=\omega^{\prime \prime}-\frac{\omega^{\prime 2}}{2 \omega}-\omega \kappa
$$

Considering the base curve $a(s)$ is decided by the null curvature $\kappa(s)$, then from (10), $X$ is determined by $\{\kappa(s), \omega(s)\}$ completely. We call the functions $\{\kappa(s), \omega(s)\}$ the structure functions of the GNS. Next, we are concerned with the relationships between the base curve $a(s)$ and the ruling flow $b(s)$ via the defined structure functions $\{\kappa(s), \omega(s)\}$.

Because $b(s)$ is a null vector field, i.e., $\langle b(s), b(s)\rangle=0$, we can regard it as a curve on the lightlike cone $\mathbb{Q}^{2}=\left\{x \in \mathbb{E}_{1}^{3} \mid\langle x, x\rangle=0\right\}$ with Cartan frame $\{b=x(\tilde{s}), \alpha(\tilde{s}), y(\tilde{s})\}$, which satisfies

$$
\left(\begin{array}{c}
\dot{x}(\tilde{s}) \\
\dot{\alpha}(\tilde{s}) \\
\dot{y}(\tilde{s})
\end{array}\right)=\left(\begin{array}{ccc}
0 & 1 & 0 \\
\tilde{\kappa}(\tilde{s}) & 0 & -1 \\
0 & -\tilde{\kappa}(\tilde{s}) & 0
\end{array}\right)\left(\begin{array}{l}
x(\tilde{s}) \\
\alpha(\tilde{s}) \\
y(\tilde{s})
\end{array}\right)
$$

and $\langle x(\tilde{s}), x(\tilde{s})\rangle=\langle y(\tilde{s}), y(\tilde{s})\rangle=\langle x(\tilde{s}), \alpha(\tilde{s})\rangle=\langle y(\tilde{s}), \alpha(\tilde{s})\rangle=0,\langle x(\tilde{s}), y(\tilde{s})\rangle=\langle\alpha(\tilde{s}), \alpha(\tilde{s})\rangle=1$, where $\tilde{s}$ is the arc length parameter of $b(\tilde{s})$ and $\tilde{\kappa}(\tilde{s})$ is the cone curvature [12]. Here the notation "." expresses the derivative with respect to $\tilde{s}$.

From (15), we have

$$
b^{\prime}(\tilde{s})=\frac{d b(\tilde{s})}{d s}=\frac{d b(\tilde{s})}{d \tilde{s}} \cdot \frac{d \tilde{s}}{d s}=\dot{x}(\tilde{s}) \frac{d \tilde{s}}{d s}=\alpha(\tilde{s}) \frac{d \tilde{s}}{d s} .
$$


Hence, from (11) and (16) together with (14), we have

$$
\alpha(\tilde{s}) \frac{d \tilde{s}}{d s}=f(s)\left(-\frac{\omega^{\prime}}{\omega} T(s)+N(s)\right) .
$$

Taking inner product on both sides of (17), from Proposition 1, we have $\left(\frac{d \tilde{s}}{d s}\right)^{2}=f^{2}(s)$. Hence $\frac{d \tilde{s}}{d s}=\epsilon f(s)(\epsilon= \pm 1)$, then

$$
\epsilon \alpha(\tilde{s})=-\frac{\omega^{\prime}}{\omega} T(s)+N(s)
$$

Differentiating (18) respect to $s$, we have from (1) and (15)

$$
\epsilon(\tilde{\kappa}(\tilde{s}) x(\tilde{s})-y(\tilde{s})) \frac{d \tilde{s}}{d s}=\left(\frac{\omega^{\prime 2}}{\omega^{2}}-\frac{\omega^{\prime \prime}}{\omega}+\kappa\right) T(s)-\frac{\omega^{\prime}}{\omega} N(s)-B(s) .
$$

Furthermore, by taking the inner product on both sides of (19), we get

$$
\tilde{\kappa}(\tilde{s}) f^{2}(s)=\frac{\omega^{\prime 2}}{2 \omega^{2}}-\frac{\omega^{\prime \prime}}{\omega}+\kappa .
$$

By (14), the cone curvature $\tilde{\kappa}(\tilde{s})$ of $b(\tilde{s})$ is given by

$$
\tilde{\kappa}(\tilde{s})=-\frac{1}{f \omega} .
$$

Hence, from (10), (19) and (21), we have

$$
y(\tilde{s})=\frac{1}{f}\left(\frac{\omega^{\prime \prime}}{\omega}-\frac{\omega^{\prime 2}}{2 \omega^{2}}-\kappa\right) T(s)=\frac{1}{\omega} T(s) .
$$

Summarizing (10), (18) and (22), we have the following conclusion.

Theorem 1. Let $X(s, t)=a(s)+t b(s)$ be a GNS with null arc length parameter of $a(s)$ in $\mathbb{E}_{1}^{3}$ and $\tilde{s}$ the arc length parameter of $b(s)$. Then the frame $\{T, N, B\}$ of $a(s)$, and the frame $\{x, \alpha, y\}$ of $b(s)$ are related by

$$
\left(\begin{array}{c}
x(\tilde{s}) \\
\epsilon \alpha(\tilde{s}) \\
y(\tilde{s})
\end{array}\right)=\left(\begin{array}{ccc}
-\frac{\omega^{\prime 2}}{2 \omega} & \omega^{\prime} & \omega \\
-\frac{\omega^{\prime}}{\omega} & 1 & 0 \\
\frac{1}{\omega} & 0 & 0
\end{array}\right)\left(\begin{array}{c}
T(s) \\
N(s) \\
B(s)
\end{array}\right)
$$

where $\epsilon=1$ for $\frac{d \tilde{s}}{d s}=f(s), \epsilon=-1$ for $\frac{d \tilde{s}}{d s}=-f(s)$.

At the same time, from (14) and (21), we have

Theorem 2. Let $X(s, t)=a(s)+t b(s)$ be a GNS with null arc length parameter of $a(s)$ in $\mathbb{E}_{1}^{3}$ and $\tilde{s}$ the arc length parameter of $b(s)$. Then the cone curvature $\tilde{\kappa}(\tilde{s})$ of $b(s)$ can be expressed by

$$
\tilde{\kappa}(\tilde{s})=\frac{2}{2 \omega^{2} \kappa+\omega^{\prime 2}-2 \omega \omega^{\prime \prime}}=-\frac{\varphi}{\omega^{2}} .
$$

Next, we focus on the geometric properties of the GNS $X$ in $\mathbb{E}_{1}^{3}$. Based on the discussion stated above, the GNS $X$ can be represented by

$$
X(s, t)=a(s)+t\left(-\frac{\omega^{\prime 2}}{2 \omega} T(s)+\omega^{\prime} N(s)+\omega B(s)\right) .
$$


Differentiating (23) with respect to $s$ and $t$, we have

$$
\left\{\begin{array}{l}
X_{s}=\left(1-t f \frac{\omega^{\prime}}{\omega}\right) T(s)+t f N(s) \\
X_{t}=-\frac{\omega^{\prime 2}}{2 \omega} T(s)+\omega^{\prime} N(s)+\omega B(s)
\end{array}\right.
$$

Then, the component functions $\{E, F, G\}$ of the first fundamental form $I$ of $X$ are

$$
\left\{\begin{array}{l}
E=\left\langle X_{s}, X_{s}\right\rangle=t^{2} f^{2} \\
F=\left\langle X_{s}, X_{t}\right\rangle=\omega \\
G=\left\langle X_{t}, X_{t}\right\rangle=0
\end{array}\right.
$$

Since $E G-F^{2}=-\omega^{2}$ and $\omega \neq 0$ everywhere, then $X$ is non-degenerate and the unit normal vector field $n$ on $X$ can be given by

$$
n(s, t)=\frac{\left(t f \frac{\omega^{\prime 2}}{2 \omega}-\omega^{\prime}\right) T(s)+\left(\omega-t f \omega^{\prime}\right) N(s)-t f \omega B(s)}{\varepsilon \omega}
$$

where $\varepsilon=1$ for $\omega>0, \varepsilon=-1$ for $\omega<0$. Since $\langle n, n\rangle=1$, we have

Theorem 3. A GNS is a timelike surface in Minkowski 3-space.

Moreover, through direct calculations, we have

$$
\left\{\begin{array}{l}
X_{s s}=t\left(f \kappa-f^{\prime} \frac{\omega^{\prime}}{\omega}-f\left(\frac{\omega^{\prime}}{\omega}\right)^{\prime}\right) T(s)+\left(1-t f \frac{\omega^{\prime}}{\omega}+t f^{\prime}\right) N(s)-t f B(s), \\
X_{s t}=f\left(-\frac{\omega^{\prime}}{\omega} T(s)+N(s)\right), \\
X_{t t}=0
\end{array}\right.
$$

Therefore, the component functions $\{L, M, N\}$ of the second fundamental form $I I$ of $X$ are given by

$$
\left\{\begin{array}{l}
L=\left\langle X_{s s}, n\right\rangle=\frac{\omega+t^{2} f^{3}+t \omega f^{\prime}-t f \omega^{\prime}}{\varepsilon \omega} \\
M=\left\langle X_{s t}, n\right\rangle=\varepsilon f \\
N=\left\langle X_{t t}, n\right\rangle=0
\end{array}\right.
$$

It is easy to get the Gaussian curvature $K$ and the mean curvature $H$ of $X$ from (25) and (28) as

$$
K=\left(\frac{f}{\omega}\right)^{2}, \quad H=\frac{\varepsilon f}{\omega} .
$$

It is readily seen that the second fundamental form $I I$ of $X$ is non-degenerate due to $X$ being non-developable. On $X$, the second fundamental form can be regarded as a new pseudo-Riemannian metric. In this case, we can define the Gaussian curvature and the mean curvature of the second fundamental form, denoted by $K_{I I}$ and $H_{I I}$ respectively.

By Briosch's formulae in Minkowski 3-space, the curvature $K_{I I}$ is called the second Gaussian curvature which is defined by [13]:

$$
K_{I I}=\frac{\left\{\left|\begin{array}{ccc}
-\frac{1}{2} L_{t t}+M_{s t}-\frac{1}{2} N_{s s} & \frac{1}{2} L_{s} & M_{s}-\frac{1}{2} L_{t} \\
M_{t}-\frac{1}{2} N_{s} & L & M \\
\frac{1}{2} N_{t} & M & N
\end{array}\right|-\left|\begin{array}{ccc}
0 & \frac{1}{2} L_{t} & \frac{1}{2} N_{s} \\
\frac{1}{2} L_{t} & L & M \\
\frac{1}{2} N_{s} & M & N
\end{array}\right|\right\}}{\left(|L N|-M^{2}\right)^{2}} .
$$

According to (28) and (30), the second Gaussian curvature $K_{I I}$ is given by

$$
K_{I I}=\frac{L_{t t}}{2 M^{2}}=\frac{\varepsilon f}{\omega} .
$$


Similarly, the curvature $H_{I I}$ is called the second mean curvature which is defined by [14]

$$
H_{I I}=H+\frac{1}{2} \Delta_{I I} \ln \sqrt{|K|},
$$

where $\Delta_{I I}$ denotes the Laplacian operator of non-degenerate second fundamental form $I I$, that is,

$$
\Delta_{I I}=-\frac{1}{\sqrt{|h|}} \sum_{i, j} \frac{\partial}{\partial x^{i}}\left(\sqrt{|h|} h^{i j} \frac{\partial}{\partial x^{j}}\right)
$$

where $\left(h_{i j}\right)$ denotes the matrix of the second fundamental form with its inverse $\left(h^{i j}\right)$, the indices $i, j$ belong to $\{1,2\}, h=\operatorname{det}\left(h_{i j}\right)$ and $\left\{x^{i}, x^{j}\right\}$ is the rectangular coordinate system $\{s, t\}$.

And by (28) and (33), the Laplacian operator of non-degenerate second fundamental form II is written by

$$
\varepsilon \Delta_{I I}=-\frac{2}{f} \frac{\partial^{2}}{\partial s \partial t}+\left(\frac{2 t f}{\omega}+\frac{f^{\prime}}{f^{2}}-\frac{\omega^{\prime}}{\omega f}\right) \frac{\partial}{\partial t}+\left(\frac{1}{f^{2}}+\frac{t^{2} f}{\omega}+\frac{t f^{\prime}}{f^{2}}-\frac{t \omega^{\prime}}{\omega f}\right) \frac{\partial^{2}}{\partial t^{2}} .
$$

From (29), (32) and (34), the second mean curvature $H_{I I}$ of $X$ is given by

$$
H_{I I}=H .
$$

From (29), (31) and (35) together with (13), we have the following result.

Theorem 4. Let $X$ be a GNS in $\mathbb{E}_{1}^{3}$. Then the Gaussian curvature $K$, the mean curvature $H$, the second Gaussian curvature $K_{I I}$, and the second mean curvature $H_{I I}$ satisfy

$$
H=K_{I I}=H_{I I}=\frac{\varepsilon}{\varphi} \quad \text { and } \quad H^{2}=K_{I I}^{2}=H_{I I}^{2}=K=\frac{1}{\varphi^{2}},
$$

where $\varphi$ is the distribution parameter of $X$.

From the above theorem, the following conclusions are straightforward.

Corollary 1. Let $X$ be a GNS in $\mathbb{E}_{1}^{3}$. Then the following statements are equivalent:

1. X has constant distribution parameters;

2. X has constant Gaussian curvature;

3. X has constant mean curvature;

4. X has constant second Gaussian curvature;

5. X has constant second mean curvature.

At the same time, since $H^{2}=K$, we have

Corollary 2. A GNS in $\mathbb{E}_{1}^{3}$ is totally umbilical.

Corollary 3. Let $X(s, t)=a(s)+t b(s)$ be a GNS in $\mathbb{E}_{1}^{3}$ with nonzero constant structure function $\omega(s)$. Then the following statements hold:

1. $X$ is congruent to a B-scroll up to a transformation;

2. $X$ is the binormal surface of its base curve $a(s)$;

3. The curvatures of $K, H, K_{I I}$ and $H_{I I}$ are all decided by the null curvature $\kappa(s)$ of $a(s)$ as follows:

$$
H^{2}=K_{I I}^{2}=H_{I I}^{2}=K=\kappa^{2} .
$$

Proof of Corollary 3. Since $\omega(s)=c$ is a nonzero constant, from (10), $X$ can be written by $X(s, t)=$ $a(s)+t c B(s),(1)$ and (2) are easily obtained. From (13), (14) and Theorem 4, the result (3) is trivial. 
Definition 3 ([7]). For a pair $\left(Y_{1}, Y_{2}\right), Y_{1} \neq Y_{2}$, of the curvatures $K, H, K_{I I}$ and $H_{I I}$ of a surface $M$ in $\mathbb{E}_{1}^{3}$, if $M$ satisfies

$$
\Phi\left(Y_{1}, Y_{2}\right)=0,
$$

Then it is said to be a $\left(Y_{1}, Y_{2}\right)$-Weingarten surface, where $\Phi$ is the Jacobi function defined by $\Phi=Y_{1} Y_{2}-Y_{2} Y_{1}$.

Because the curvatures $K, H, K_{I I}$ and $H_{I I}$ are all functions of $s$, a GNS $X$ satisfies Jacobian conditions $\Phi\left(Y_{1}, Y_{2}\right)=0$, obviously. Then we have

Theorem 5. A GNS in $\mathbb{E}_{1}^{3}$ is $\left(Y_{1}, Y_{2}\right)$-Weingarten surface, where $\left\{Y_{1}, Y_{2}\right\}=\left\{K, H, K_{I I}, H_{I I}\right\}$ and $Y_{1} \neq Y_{2}$.

\section{Special Generalized Null Scrolls}

In this section, let us characterize the GNS whose base curve is a k-type null helix in $\mathbb{E}_{1}^{3}$.

Definition 4 ([15]). Let $r(s)$ be a null curve framed by $\{T, N, B\}$ in $\mathbb{E}_{1}^{3}$. If there exists a nonzero constant vector field $V \in \mathbb{E}_{1}^{3}$ such that $\langle T, V\rangle \neq 0(\langle N, V\rangle \neq 0,\langle B, V\rangle \neq 0$, respectively $)$ is a constant, then it is said to be a $k$-type $(k=1,2,3$, respectively) null helix and $V$ is called the axis of $r(s)$.

Remark 3 ([15]). For a null curve with null curvature $\kappa(s)$, it is a 1-type or 3-type null helix if and only if $\kappa(s)$ is a constant and it is a 2-type null helix if and only if $\kappa(s)=\frac{c_{1}}{\left(s+c_{2}\right)^{2}}$, where $c_{1} \neq 0$ and $c_{2}$ are constants.

Proposition 3. Let $X(s, t)=a(s)+t b(s)$ be a GNS in $\mathbb{E}_{1}^{3}$ and its base curve a (s) is a 1-type (or 3-type) null helix. Then the GNS can be expressed by

$$
X(s, t)=a_{i}(s)+t b_{i}(s),(i=1,2,3)
$$

where $a_{i}(s)$ and $b_{i}(s)$ are stated in (37)-(42).

Proof of Proposition 3. When the base curve $a(s)$ is a 1-type or 3-type null helix, the null curvature $\kappa=\kappa(s)$ is a constant. Let $\kappa= \pm \frac{c^{2}}{2},(0 \leq c \in \mathbb{R})$, by (2)

$$
\kappa=\frac{1}{2}\left[\left(\log g^{\prime}\right)^{\prime}\right]^{2}-\left(\log g^{\prime}\right)^{\prime \prime}= \pm \frac{c^{2}}{2} .
$$

Solving the above Riccati Equation (36), the structure function $g(s)$ of $a(s)$ is as follows:

1. When $\kappa=-\frac{c^{2}}{2}<0, g(s)=\frac{2}{c} \tan \left(\frac{c s}{2}\right)$;

2. When $\kappa=c=0, g(s)=-\frac{a}{s}, a \in \mathbb{R}-\{0\}$;

3. When $\kappa=\frac{c^{2}}{2}>0, g(s)=\frac{2}{c} \tanh \left(\frac{\mathcal{C S}}{2}\right)$.

Substituting the above conclusions into (3) together with (10), through direct calculations, the base curve $a_{i}(s)$ and the ruling $b_{i}(s)(i=1,2,3)$ can be expressed by:

1. When $\kappa=-\frac{c^{2}}{2}<0$, we have

$$
\begin{gathered}
a_{1}(s)=\left(\frac{4-c^{2}}{4 c^{2}} s-\frac{4+c^{2}}{4 c^{3}} \sin c s,-\frac{1}{c^{2}} \cos c s, \frac{4+c^{2}}{4 c^{2}} s-\frac{4-c^{2}}{4 c^{3}} \sin c s\right) \\
b_{1}(s)=-\frac{\omega^{\prime 2}}{2 \omega} T_{1}+\omega^{\prime} N_{1}+\omega B_{1}
\end{gathered}
$$


where

$$
\left\{\begin{array}{l}
T_{1}=\left(\frac{2}{c^{2}} \sin ^{2} \frac{c s}{2}-\frac{1}{2} \cos ^{2} \frac{c s}{2}, \frac{1}{c} \sin c s, \frac{2}{c^{2}} \sin ^{2} \frac{c s}{2}+\frac{1}{2} \cos ^{2} \frac{c s}{2}\right), \\
N_{1}=\left(\frac{4+c^{2}}{4 c} \sin c s, \cos c s, \frac{4-c^{2}}{4 c} \sin c s\right) \\
B_{1}=\left(\frac{c^{2}}{4} \sin ^{2} \frac{c s}{2}-\cos ^{2} \frac{c s}{2}, \frac{c}{2} \sin c s,-\frac{c^{2}}{4} \sin ^{2} \frac{c s}{2}-\cos ^{2} \frac{c s}{2}\right) .
\end{array}\right.
$$

2. When $\kappa=c=0$, we have

$$
\begin{aligned}
& a_{2}(s)=\left(\frac{a s}{2}-\frac{s^{3}}{6 a},-\frac{s^{2}}{2}, \frac{a s}{2}+\frac{s^{3}}{6 a}\right), \\
& b_{2}(s)=-\frac{\omega^{\prime 2}}{2 \omega} T_{2}+\omega^{\prime} N_{2}+\omega B_{2},
\end{aligned}
$$

where

$$
\left\{\begin{array}{l}
T_{2}=\left(\frac{a}{2}-\frac{s^{2}}{2 a},-s, \frac{a}{2}+\frac{s^{2}}{2 a}\right), \\
N_{2}=\left(-\frac{s}{a},-1, \frac{s}{a}\right), \\
B_{2}=\left(\frac{1}{a}, 0,-\frac{1}{a}\right) .
\end{array}\right.
$$

3. When $\kappa=\frac{c^{2}}{2}>0$, we have

$$
\begin{gathered}
a_{3}(s)=\left(\frac{4-c^{2}}{4 c^{3}} \sinh c s-\frac{4+c^{2}}{4 c^{2}} s, \frac{1}{c^{2}} \cosh c s, \frac{4+c^{2}}{4 c^{3}} \sinh c s-\frac{4-c^{2}}{4 c^{2}} s\right), \\
b_{3}(s)=-\frac{\omega^{\prime 2}}{2 \omega} T_{3}+\omega^{\prime} N_{3}+\omega B_{3},
\end{gathered}
$$

where

$$
\left\{\begin{array}{l}
T_{3}=\left(\frac{2}{c^{2}} \sinh ^{2} \frac{c s}{2}-\frac{1}{2} \cosh ^{2} \frac{c s}{2}, \frac{1}{c} \sinh c s, \frac{2}{c^{2}} \sinh ^{2} \frac{c s}{2}+\frac{1}{2} \cosh ^{2} \frac{c s}{2}\right), \\
N_{3}=\left(\frac{4-c^{2}}{4 c} \sinh c s, \cosh c s, \frac{4+c^{2}}{4 c} \sinh c s\right), \\
B_{3}=\left(\frac{c^{2}}{4} \sinh ^{2} \frac{c s}{2}-\cosh ^{2} \frac{c s}{2},-\frac{c}{2} \sinh c s,-\frac{c^{2}}{4} \sinh ^{2} \frac{c s}{2}-\cosh ^{2} \frac{c s}{2}\right) .
\end{array}\right.
$$

From the expression of $a_{i}(s)$ and $b_{i}(s)$ stated above, the results can be achieved.

From Proposition 3 and Corollary 3, the following result is obvious.

Corollary 4. Let $X(s, t)=a(s)+t b(s)$ be a GNS in $\mathbb{E}_{1}^{3}$ with nonzero constant structure functions $\omega(s)$ and the base curve is a 1-type (or 3-type) null helix with null curvature $k(s) \neq 0$. Then, the GNS can be written by 1. When $\kappa=-\frac{c^{2}}{2}(0<c \in \mathbb{R}), \omega=a(0 \neq a \in \mathbb{R}), X=a(s)+a t b(s)$. Where

$$
a(s)=\left(\frac{4-c^{2}}{4 c^{2}} s-\frac{4+c^{2}}{4 c^{3}} \sin c s,-\frac{1}{c^{2}} \cos c s, \frac{4+c^{2}}{4 c^{2}} s-\frac{4-c^{2}}{4 c^{3}} \sin c s\right)
$$

and

$$
b(s)=\left(\frac{c^{2}}{4} \sin ^{2} \frac{c s}{2}-\cos ^{2} \frac{c s}{2}, \frac{c}{2} \sin c s,-\frac{c^{2}}{4} \sin ^{2} \frac{c s}{2}-\cos ^{2} \frac{c s}{2}\right) ;
$$

2. When $\kappa=\frac{c^{2}}{2}(0<c \in \mathbb{R}), \omega=a(0 \neq a \in \mathbb{R}), X=a(s)+a t b(s)$. Where

$$
a(s)=\left(\frac{4-c^{2}}{4 c^{3}} \sinh c s-\frac{4+c^{2}}{4 c^{2}} s, \frac{1}{c^{2}} \cosh c s, \frac{4+c^{2}}{4 c^{3}} \sinh c s-\frac{4-c^{2}}{4 c^{2}} s\right),
$$

and

$$
b(s)=\left(\frac{c^{2}}{4} \sinh ^{2} \frac{c s}{2}-\cosh ^{2} \frac{c s}{2},-\frac{c}{2} \sinh c s,-\frac{c^{2}}{4} \sinh ^{2} \frac{c s}{2}-\cosh ^{2} \frac{c s}{2}\right) .
$$


Example 1. Let $X=X(s, t)$ be a GNS in Proposition 3 with $\omega(s)=e^{s}$. Then

- when $\kappa=-\frac{1}{2}$, X can be written by (see Figure 1)

$$
X=\left(\frac{3}{4} s-\frac{5}{4} \sin s,-\cos s, \frac{5}{4} s-\frac{3}{4} \sin s\right)+t e^{s}\left(\frac{5}{4} \sin s-\frac{3}{4}, \cos s, \frac{3}{4} \sin s-\frac{5}{4}\right) ;
$$

- when $\kappa=0$ and $a=1, X$ can be expressed by (see Figure 2)

$$
X=\left(\frac{1}{2} s-\frac{1}{6} s^{3},-\frac{1}{2} s^{2}, \frac{1}{2} s+\frac{1}{6} s^{3}\right)+t e^{s}\left(\frac{1}{4} s^{2}-s+\frac{3}{4}, \frac{1}{2} s-1,-\frac{1}{4} s^{2}+s+\frac{3}{4}\right) ;
$$

- when $\kappa=\frac{1}{2}$, X can be written by (see Figure 3)

$$
X=\left(\frac{3}{4} \sinh s-\frac{5}{4} s, \cosh s, \frac{5}{4} \sinh s-\frac{3}{4} s\right)+t e^{s}\left(\frac{3}{4} \sinh s-\frac{3}{4} \cosh s, \cosh s-\sinh s, \frac{5}{4} \sinh s-\frac{5}{4} \cosh s\right) .
$$

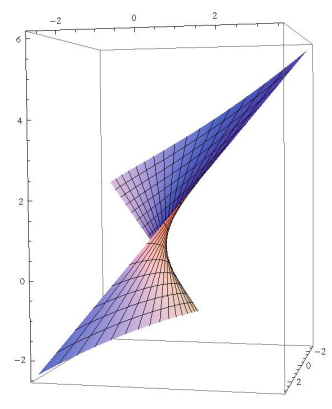

Figure 1. GNS for $\kappa=-\frac{1}{2}$.

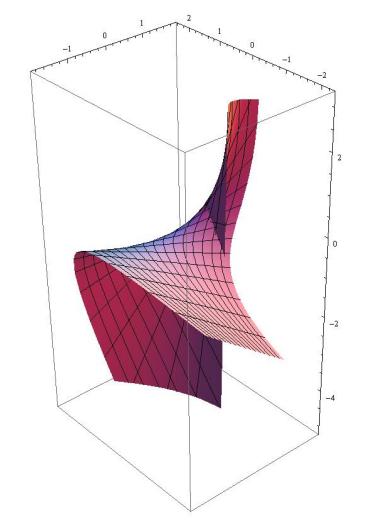

Figure 2. GNS for $\kappa=0$.

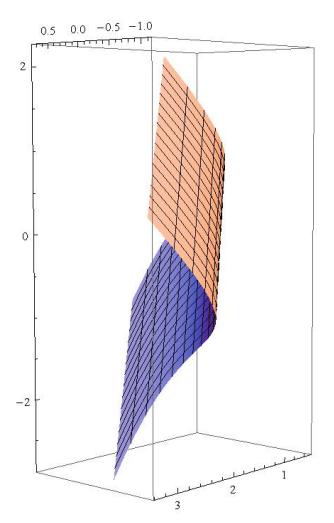

Figure 3. GNS for $\kappa=\frac{1}{2}$. 
Proposition 4. Let $X(s, t)=a(s)+t b(s)$ be a GNS in $\mathbb{E}_{1}^{3}$ and its base curve a(s) is a 2-type null helix. Then the GNS can be expressed by

$$
X=a_{i}(s)+t b_{i}(s),(i=1,2,3)
$$

where $a_{i}(s)$ and $b_{i}(s)$ are stated in (45)-(50).

Proof of Proposition 4. When the base curve $a(s)$ is a 2-type null helix, the null curvature $\kappa(s)$ is $\frac{1}{2} c_{1}\left(s+c_{2}\right)^{-2}$, where $c_{1} \neq 0$ and $c_{2}$ are constants. By an appropriate parameter transformation and from (2), we have

$$
\kappa=\frac{1}{2}\left[\left(\log g^{\prime}\right)^{\prime}\right]^{2}-\left(\log g^{\prime}\right)^{\prime \prime}=\frac{c_{1}}{2 s^{2}} .
$$

Solving the above differential Equation (44), the structure functions $g(s)$ of $a(s)$ are as follows:

1. $g(s)=s^{c}$ or $g(s)=s^{-c}$, for $c \neq 0, \pm 1$ and $c_{1}=c^{2}-1$;

2. $g(s)=\frac{c}{\log s}$ or $g(s)=\frac{\log s}{c}$, for $c \neq 0, \pm 1$ and $c_{1}=-1$;

3. $g(s)=\frac{2}{c} \tan \left(\frac{c}{2} \log s\right)$ or $g(s)=-\frac{2}{c} \tan ^{-1}\left(\frac{c}{2} \log s\right)$, for $c \neq 0$ and $c_{1}=-c^{2}-1$.

Similar to Proposition 3, the base curve $a_{i}(s)$ and the ruling $b_{i}(s)(i=1,2,3)$ can be represented by

1. When $c_{1}=c^{2}-1,(c \neq 0, \pm 1)$, we have

$$
\begin{gathered}
a_{1}(s)=\frac{1}{2 c}\left(\frac{s^{c+2}}{c+2}-\frac{s^{2-c}}{2-c}, s^{2}, \frac{s^{c+2}}{c+2}+\frac{s^{2-c}}{2-c}\right), \\
b_{1}(s)=-\frac{\omega^{\prime 2}}{2 \omega} T_{1}+\omega^{\prime} N_{1}+\omega B_{1},
\end{gathered}
$$

where

$$
\left\{\begin{array}{l}
T_{1}=\frac{s}{2 c}\left(s^{c}-s^{-c}, 2, s^{c}+s^{-c}\right), \\
N_{1}=\frac{1}{2 c}\left((c+1) s^{c}+(c-1) s^{-c}, 2,(c+1) s^{c}-(c-1) s^{-c}\right), \\
B_{1}=\frac{1}{4 c}\left(-(c+1)^{2} s^{c-1}+(c-1)^{2} s^{-c-1}, \frac{2\left(c^{2}-1\right)}{s} \cdot-(c+1)^{2} s^{c-1}-(c-1)^{2} s^{-c-1}\right) .
\end{array}\right.
$$

2. When $c_{1}=-1$, we have

$$
\begin{gathered}
a_{2}(s)=\frac{s^{2}}{4}\left(c-\frac{\log ^{2} s}{c}+\frac{\log s}{c}-\frac{1}{2 c}, 2 \log s-1, c+\frac{\log ^{2} s}{c}-\frac{\log s}{c}+\frac{1}{2 c}\right), \\
b_{2}(s)=-\frac{\omega^{\prime 2}}{2 \omega} T_{2}+\omega^{\prime} N_{2}+\omega B_{2},
\end{gathered}
$$

where

$$
\left\{\begin{array}{l}
T_{2}=\frac{s \log s}{2}\left(\frac{c}{\log s}-\frac{\log s}{c}, 2, \frac{c}{\log s}+\frac{\log s}{c}\right), \\
N_{2}=\frac{1}{2}\left(c-\frac{\log ^{2} s}{c}-\frac{2 \log s}{c}, 2 \log s+2, c+\frac{\log ^{2} s}{c}+\frac{2 \log s}{c}\right), \\
B_{2}=\left(\frac{\log ^{2} s}{4 c s}+\frac{1+\log s}{c s}-\frac{c}{4 s},-\frac{2+\log s}{2 s},-\frac{\log ^{2} s}{4 c s}-\frac{1+\log s}{c s}-\frac{c}{4 s}\right) .
\end{array}\right.
$$


3. When $c_{1}=-c^{2}-1$, we have

$$
\begin{gathered}
a_{3}(s)=\left(\frac{s^{2}}{8 c^{2}}\left(4-c^{2}-2 c \sin (c \log s)-4 \cos (c \log s)\right), \frac{s^{2}}{c^{2}+4}\left(\frac{2}{c} \sin (c \log s)-\cos (c \log s)\right),\right. \\
\left.\frac{s^{2}}{8 c^{2}}\left(4+c^{2}-\frac{2 c\left(4-c^{2}\right)}{4+c^{2}} \sin (c \log s)-\frac{4\left(4-c^{2}\right)}{4+c^{2}} \cos (c \log s)\right)\right), \\
b_{3}(s)=-\frac{\omega^{\prime 2}}{2 \omega} T_{3}+\omega^{\prime} N_{3}+\omega B_{3},
\end{gathered}
$$

where

$$
\left\{\begin{aligned}
T_{3}= & \frac{s}{c}\left(\frac{4-c^{2}}{4 c}-\frac{4+c^{2}}{4 c} \cos (c \log s), \sin (c \log s), \frac{4+c^{2}}{4 c}-\frac{4-c^{2}}{4 c} \cos (c \log s)\right) \\
N_{3}= & \left(\frac{4-c^{2}}{4 c^{2}}-\frac{4+c^{2}}{4 c^{2}}(\cos (c \log s)+c \sin (c \log s)), \frac{\sin (c \log s)+c \cos (c \log s)}{c}\right. \\
& \left.\frac{4+c^{2}}{4 c^{2}}-\frac{4-c^{2}}{4 c^{2}}(\cos (c \log s)+c \sin (c \log s))\right) \\
B_{3}= & \left(\frac{\left(c^{2}+4\right)\left[\left(3 c^{2}+1\right) \cos (c \log s)-2 c \sin (c \log s)\right]+\left(c^{2}+1\right)\left(c^{2}-4\right)}{8 c^{2} s}\right. \\
& \frac{\left(c^{2}-1\right) \sin (c \log s)-2 c \cos (c \log s)}{2 c s} \\
& \left.\frac{\left(4-c^{2}\right)\left[\left(3 c^{2}+1\right) \cos (c \log s)-2 c \sin (c \log s)\right]+\left(c^{2}+1\right)\left(c^{2}+4\right)}{8 c^{2} s}\right)
\end{aligned}\right.
$$

From the expression of $a_{i}(s)$ and $b_{i}(s)$ stated above, the results can be obtained.

Example 2. Let $X=X(s, t)$ be a GNS in Proposition 4.

- Assume $\omega(s)=-\frac{1}{4} s^{2}, \kappa=\frac{4}{s^{2}}$, then $X$ can be expressed by (see Figure 4)

$$
X=\left(\frac{s^{5}}{30}+\frac{1}{6 s}, \frac{s^{2}}{6}, \frac{s^{5}}{30}-\frac{1}{6 s}\right)+t\left(\frac{s^{4}}{12}-\frac{1}{3 s^{2}},-\frac{s}{3}, \frac{s^{4}}{12}+\frac{1}{3 s^{2}}\right) .
$$

- Assume $\omega(s)=2 s^{2}, \kappa=-\frac{1}{2 s^{2}}$, then X can be obtained as (see Figure 5)

$$
\begin{aligned}
X= & \frac{1}{4} s^{2}\left(\log ^{2} s-\log s-\frac{1}{2}, 2 \log s-1,-\log ^{2} s+\log s-\frac{3}{2}\right)+ \\
& t\left(-\frac{s \log ^{2} s}{2}+2 s \log s-\frac{3}{2} s, 2 s-s \log s, \frac{s \log ^{2} s}{2}-2 s \log s+\frac{5}{2} s\right) .
\end{aligned}
$$

- Assume $\omega(s)=8, \kappa=-\frac{1}{s^{2}}$, then $X$ can be written by (see Figure 6)

$$
\begin{aligned}
X= & \frac{s^{2}}{40}(15-10 \sin (\log s)-20 \cos (\log s), 16 \sin (\log s)-8 \cos (\log s), \\
& 25-6 \sin (\log s)-12 \cos (\log s))+\frac{t}{s}(20 \cos (\log s)-10 \sin (\log s)-6,-8 \cos (\log s), \\
& 12 \cos (\log s)-6 \sin (\log s)+10) .
\end{aligned}
$$

Remark 4. Through the above two examples, we have presented a method to characterize GNS by the defined structure functions explicitly. This work can be generalized to a large class of surfaces and applied into CAD (CAGD) or product design etc. 


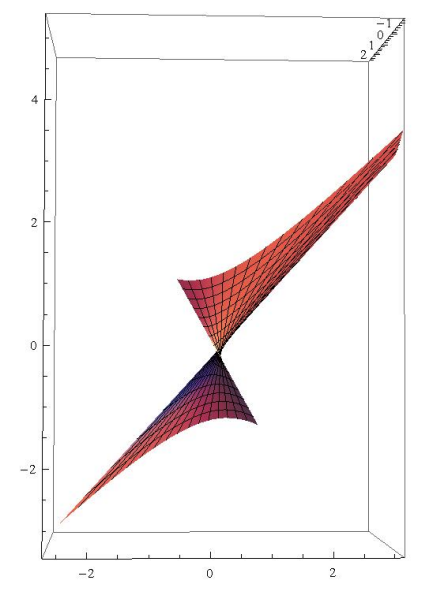

Figure 4. GNS for $\omega=-\frac{s^{2}}{4}$ and $\kappa=\frac{4}{s^{2}}$.

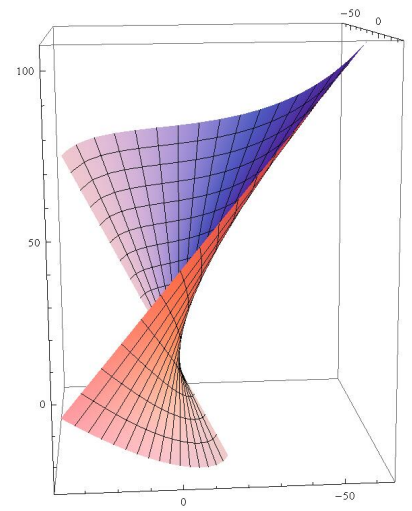

Figure 5. GNS for $\omega=2 s^{2}$ and $\kappa=-\frac{1}{2 s^{2}}$.

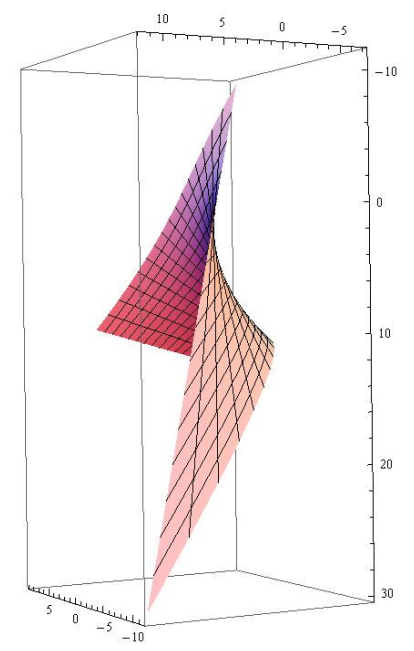

Figure 6. GNS for $\omega=8$ and $\kappa=-\frac{1}{s^{2}}$.

Author Contributions: J.Q. and X.F. set up the problem and computed the details. S.D.J. checked and polished the draft.

Funding: The first author was supported by NSFC (No.11801065,11371080) and the third author was supported by the National Research Foundation of Korea (NRF) grant funded by the Korea government (MSIP) (NRF-2018R1A2B2002046).

Acknowledgments: We thank the referee for the careful review and the valuable comments to improve the paper.

Conflicts of Interest: The authors declare no conflict of interest. 


\section{References}

1. Kim, Y.H.; Yoon, D.W. On non-developable ruled surface in Lorentz-Minkowski 3-spaces. Taiwan J. Math. 2007, 11, 197-214. [CrossRef]

2. Kim, Y.H.; Yoon, D.W. Classification of ruled surfaces in Minkowski 3-spaces. J. Geom. Phys. 2004, 49, 89-100. [CrossRef]

3. Barros, M.; Ferrandez, A. How big is the family of stationary null scrolls? J. Geom. Phys. 2013, 64, 54-60. [CrossRef]

4. Choi, S.M.; Ki, U.H.; Suh, Y.J. On the Gauss map of null scrolls. Tsukuba J. Math. 1998, 22, 273-279. [CrossRef]

5. Pak, J.S.; Yoon, D.W. On null scrolls satisfying the condition $\Delta H=A H$. Commun. Korean Math. Soc. 2000, 15, 533-540.

6. Graves, L.K. Codimension one isometric immersions between Lorentz spaces. Trans. Am. Math. Soc. 1979, 252, 367-392. [CrossRef]

7. Kim, Y.H. Differential geometry of generalized B-scroll and II-Weingarten surfaces of Minkowski space. Proc. Natl. Inst. Math. Sci. 2006, 6, 67-76.

8. Qian, J.; Kim, Y.H. Directional associated curves of a null curve in $\mathbb{E}_{1}^{3}$. Bull. Korean Math. Soc. 2015, 52, 183-200. [CrossRef]

9. Ferrandez, A.; Gimenez, A.; Lucas, P. Null helices in Lorentzian space form. Int. J. Mod. Phys. A 2001, 12, 4845-4863. [CrossRef]

10. Inoguchi, J.; Lee, S. Null curves in Monkowski 3-space. Int. Electron. J. Geom. 2008, 1, 40-83.

11. Yayli, Y. On the motion of the Frenet vectors and spacelike ruled surfaces in the Minkowski 3-space . Math. Comput. Appl. 2000, 5, 49-55. [CrossRef]

12. Liu, H. Curves in the lightlike cone. Contrib. Algebra Geom. 2004, 45, 291-303.

13. Blair, D.E.; Koufogiorgos, T. Ruled surfaces with vanishing second Gaussian curvature. Monatshefte Math. 1992, 113, 177-181. [CrossRef]

14. Dillen, F.; Sodsiri, W. Ruled surfaces of Weingarten type in Minkowski 3-space. J. Geom. 2005, 83, $10-21$. [CrossRef]

15. Qian, J.; Liu, H.; Jin, G. Lightlike Helices in 3D Minkowski Space. J. Northeast. Univ. (Nat. Sci.) 2012, 33, 1800-1802.

(C) 2019 by the authors. Licensee MDPI, Basel, Switzerland. This article is an open access article distributed under the terms and conditions of the Creative Commons Attribution (CC BY) license (http:/ / creativecommons.org/licenses/by/4.0/). 